\title{
Results from the ROSAT EUV Wide Field Camera
}

\author{
JOHN P. PYE
}

Department of Physics \& Astronomy, Leicester University, Leicester, LE1 7RH, UK

I review some of the major achievements of the ROSAT extreme-ultraviolet all-sky survey, including the results of the recently completed $2 \mathrm{RE}$ source catalogue and associated study of temporal variability in EUV sources.

\section{Introduction}

In January 1989, at the first Berkeley colloquium on extreme-ultraviolet (EUV) astronomy, I reviewed, prelaunch, the expected performance and calibration parameters of the ROSAT EUV Wide Field Camera (Pye et al. 1991), and predicted numbers of sources of different classes that should be detectable in the all-sky survey. So it is timely now, six years later at this second Berkeley colloquium on EUV astronomy, to summarise the achievements of the WFC, especially those from the all-sky survey.

During 1990-1991 the WFC performed the first all-sky survey at EUV wavelengths. The survey was conducted in two 'colours' using broad-band filters to define wavebands covering the ranges $60-140 \AA$ and $112-200 \AA$. It was fully imaging, with effective spatial resolution of about 3 arcmin FWHM, and point source location accuracy of typically better than 1 arcmin. From an initial analysis, Pounds et al. (1993) published the WFC Bright Source Catalogue (BSC) of 383 sources. They also assessed why, for the two main classes of EUV source, there were substantially fewer detections than predicted prelaunch. Briefly, for white-dwarf stars (WDs), the hotter objects $\left(T_{\text {eff }} \gtrsim 4 \times 10^{4} \mathrm{~K}\right)$ have their EUV (and soft X-ray) luminosities greatly reduced by the opacity of trace metals radiatively levitated in the WD atmospheres (Barstow et al. 1993). For the coronal emission from late-type $(\mathrm{F}-\mathrm{M})$ stars the explanation appears to lie, at least in part, with over-estimation of the high-luminosity tails in early X-ray luminosity functions, especially for dM stars. The sensitivity of flux/count-rate conversion factor to the assumed (and rather ill-defined) source spectra, may also be a significant cause of error (Hodgkin \& Pye 1994).

\section{2RE Catalogue}

Reprocessing of the complete survey database has resulted in a new catalogue of EUV sources, designated '2RE' (Pye et al. 1995). The $2 \mathrm{RE}$ Catalogue contains 479 sources (see figure 1), 120 of which were not reported in the BSC; of these, 97 also do not appear in the first $E U V E$ Catalogue (Bowyer et al. 1994) and hence have not previously been reported at EUV wavelengths. There are $3872 \mathrm{RE}$ sources detected in both survey wavebands, a significant advance on the BSC ( 80 percent versus 60 percent). Improvements over the original BSC include: (i) better rejection of poor aspect periods, and smaller random errors in the aspect reconstruction; (ii) improved background screening; (iii) improved methods for source detection; (iv) inclusion of a time-variability test for each source; (v) more extensive investigation of the survey sensitivity. Most sources (444 out of 479) have proposed identifications; these include 75 with late-type stars and 6 with hot white-dwarf stars, amongst the 120 new, 'post-BSC' sources. Overall, as with the BSC, 


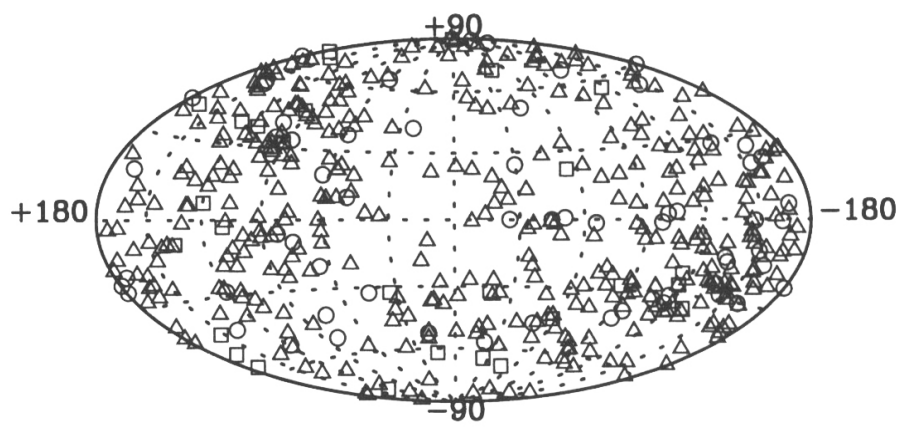

Figure 1. Map of the sky in galactic coordinates, showing the locations of the EUV sources in the 2RE Catalogue. The projection is Hammer equal area. Sources detected in both S1 and S2 wavebands are indicated by triangles, while those detected only in S1 or S2 are marked with circles and squares respectively.

the main constituents of the 2RE Catalogue are, in order, late-type stars (F-M), hot white-dwarf stars, and cataclysmic variables (see figure 2).

\section{Temporal Variability}

Light-curves for each 2RE source were generated and tested for constancy on timescales $\sim 1.5 \mathrm{~h}$ (the scanning-survey sampling interval). As a result, 31 sources were classified as variable (McGale et al. 1995a,b). Cataclysmic variables (CVs) were found to be the most active group with about 50 percent of them displaying variability, compared with about 10 percent of late-type stars. All the variable G-type stars are known or possible RS CVn-type binaries; $~ 40$ percent of the 2RE G-type RS CVn systems are found to vary. Other variable sources are the high-mass X-ray binary Her X-1, the eclipsing binaries Algol (B8 V + K0 IV) and V471 Tau (DA + K2 V) and the active galaxy Mkn 478.

One of the most extreme examples of EUV variability seen to date, and certainly a highlight of the WFC pointed observation programme, is the EUV transient source RE J1255+266 (see Dahlem et al. 1995a; also Dahlem \& Kreysing 1994; Dahlem et al. $1995 \mathrm{~b}$ ). It has been identified optically as a binary system consisting of a DA white dwarf with a low-mass companion (Watson et al. 1995).

\section{Optical Identification Programme}

Mason et al. (1995) have reported the results of the WFC survey optical identification programme. This programme, which concentrated on BSC sources without secure catalogue counterparts, produced identifications in 195 of the 216 EUV source fields observed. The newly identified EUV emitters include 69 new white dwarfs, 114 active stars, 7 new magnetic cataclysmic variables and 5 active galaxies, 4 of which are newly 


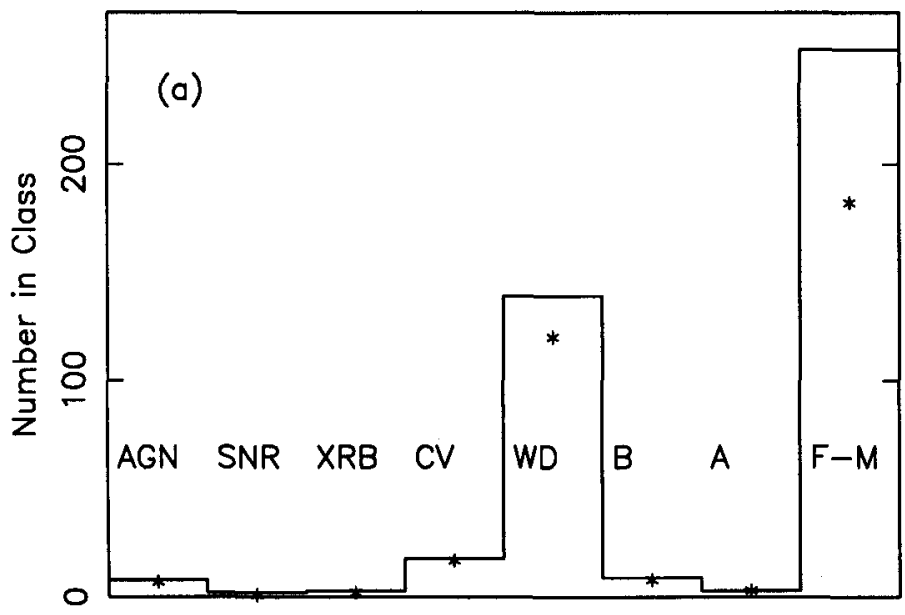

Identification Class

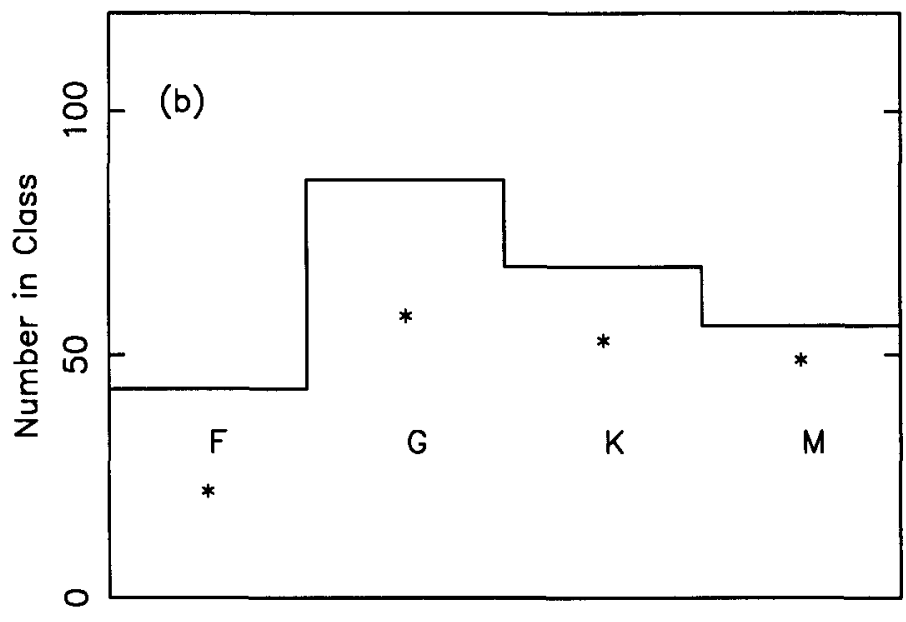

Identification Class

Figure 2. (a) Distribution of optical counterparts in the 2RE Catalogue. The star symbols show the same information, but for the BSC (figure 4a of Pounds et al. 1993). (b) Distribution of identifications in the main subgroup of late-type stars. 
identified. Several of the white dwarfs identified are in non-interacting binaries, one CV (2RE J0751+144) uniquely appears to share characteristics of the both the Polars and the DQ Her-type CVs, and one of the AGN (2RE J1034+393) has the highest EUV/UV flux ratio known.

\section{Other Survey Studies}

Based on the BSC or $2 \mathrm{RE}$, and on the associated data products (images, light-curves, photon event lists, etc.), there have been various detailed investigations of specific topics and classes of source. Many references to papers on such studies and on individual sources, can be found in Pounds et al. (1993) and Pye et al. (1995). Descriptions of the instrument, its performance and calibration can be found in Barstow \& Willingale (1988), Sims et al. (1990), Pye et al. (1991), Wells et al. (1990), Pounds et al. (1993) and Pye et al. (1995), and references therein.

\section{On-Line Access to the Survey Archive}

The 2RE Catalogue, and other survey data products such as images, are available on-line via Internet, at the Leicester University Database and Archive Service (LEDAS). LEDAS can be accessed by telnet (ledas.star.le.ac.uk), anonymous ftp (ledas-ftp.star.le. ac.uk) and WWW (URL: http://ledas-www.star.le.ac.uk). The WFC survey also has its own WWW page (accessible from the LEDAS home page):

http://ledas-www.star.le.ac.uk/rosat_euv.

ROSAT is a collaborative project between Germany, the UK and USA. I am happy to acknowledge the great efforts of many colleagues in the ROSAT Project in ensuring the successful operation of the WFC. The UK ROSAT Project is funded by the Particle Physics and Astronomy Research Council.

\section{REFERENCES}

Barstow, M. A. \& Willingale, R. 1988, JBIS., 41, 345

BARstow, M. A., ET AL. 1993, MNRAS, 264, 16

Bowyer, S., Lieu, R., Lampton, M., Lewis, J., Wu, X., Drake, J. J., \& Malina, R. F. 1994, ApJS., 93, 569

Dahlem, M. \& Kreysing, H. -C. 1994, IAU Circular No. 6085

DAHLEM, M., ET AL. 1995a, these proceedings

Dahlem, M., Kreysing, H. -C., White, S., Engels, D., Condon, J. J., \& Voges, W. $1995 \mathrm{~b}, \mathrm{~A} \& \mathrm{~A}$, in press

McGale, P. A., Pye, J. P., Barber, C. R., \& Page, C. G. 1995a, these proceedings

McGale, P. A., Pye, J. P., Barber, C. R., \& Page, C. G. 1995b, MNRAS, in press

MASON, K. O., ET AL. 1995, MNRAS, 274, 1194

Pounds, K. A., ET AL. 1993, MNRAS., 260, 77

Pye, J. P., Watson, M. G., Pounds, K. A., \& Wells, A. 1991, in Extreme Ultraviolet Astronomy, ed. R. F. Malina \& S. Bowyer, 409, New York: Pergamon

PYe, J. P., ET AL. 1995, MNRAS, 274, 1165

Sims, M. R. ET AL. 1990, Opt. Eng., 29, 649

Watson, M. G., McMahon, R. G., \& Page, M. J. 1995, IAU Circular No. 6126

Wells, A., ET AL. 1990, Proc. SPIE, 1344, 230 